\title{
Author Correction: Characterizing rare and low-frequency height-associated variants in the Japanese population
}

\author{
Masato Akiyama, Kazuyoshi Ishigaki, Saori Sakaue, Yukihide Momozawa, Momoko Horikoshi, Makoto Hirata (D), \\ Koichi Matsuda (D), Shiro Ikegawa (D), Atsushi Takahashi (D), Masahiro Kanai (D), Sadao Suzuki, Daisuke Matsui, \\ Mariko Naito, Taiki Yamaji, Motoki Iwasaki, Norie Sawada (B), Kozo Tanno, Makoto Sasaki (D, Atsushi Hozawa, \\ Naoko Minegishi, Kenji Wakai, Shoichiro Tsugane (D), Atsushi Shimizu, Masayuki Yamamoto, Yukinori Okada (D), \\ Yoshinori Murakami (1), Michiaki Kubo \& Yoichiro Kamatani (i)
}

Correction to: Nature Communications https://doi.org/10.1038/s41467-019-12276-5, published online 27 September 2019.

In the original version of this paper, under the subheading "Genome-wide association study" in the last-but-one sentence, the number of variants included in the analysis of overlap between GWAS variants in the BBJ and in European population GWAS was incorrectly given as 1416, leading to incorrect association results. The correct number of analysed variants is 1417 and the sentences should read "Of these variants, 1417 variants were evaluated in our GWAS (Supplementary Data 6), and 772 variants were nominally associated $\left(P_{\mathrm{GWAS}}<0.05\right)$ with the consistent direction of effects. These 772 variants represented a significant positive correlation in the effect sizes between our GWAS and the meta-analysis of GIANT and UKB (Pearson's $r=0.82, P=7.00 \times 10^{-189}$ ), suggesting that these are shared height-associated variants across populations."

In addition, the list of variants in Supplementary Data 6 ("Associations of Japanese GWAS at reported variants in large-scale European GWAS”) was incorrect. Two out of 1417 evaluated variants were inadvertently omitted from the table while 460 variants were included by mistake (based on overlap of BBJ GWAS with 3290 lead variants from European GWAS instead of 2481 variants from the nonconditioned analysis, which would have been correct). The corrected Supplementary Data 6 lists the correct 1417 variants obtained in this analysis.

Finally, there was a typo in the methods section, under the subheading "Genotyping and imputation" in the fourth sentence. The sentence originally read "We further compared the genotypes of the overlapping variants between the GWAS array and WGS and excluded variants with a concordance rate $<99.5 \%$ or a non-reference concordance rate $\geq 0.5 \%$ " where the second "concordance" (in "non-reference concordance rate $\geq 0.5 \%$ ") should have been "discordance".

All of these errors have now been corrected in the HTML and PDF versions of the article and the Supplementary Data 6 file has been updated in the HTML version of the article.

Published online: 09 March 2020

Open Access This article is licensed under a Creative Commons Attribution 4.0 International License, which permits use, sharing, adaptation, distribution and reproduction in any medium or format, as long as you give appropriate credit to the original author(s) and the source, provide a link to the Creative Commons license, and indicate if changes were made. The images or other third party material in this article are included in the article's Creative Commons license, unless indicated otherwise in a credit line to the material. If material is not included in the article's Creative Commons license and your intended use is not permitted by statutory regulation or exceeds the permitted use, you will need to obtain permission directly from the copyright holder. To view a copy of this license, visit http://creativecommons.org/licenses/by/4.0/. 